NEW PPE MASK RANGE BEGINS KILLING SARSCOV-2 ON CONTACT

Lancaster based ViraCoat Limited has announced the global launch of ViraCoat 3 Ply Type 2R Surgical Face Mask, ViraCoat FFP2 Respirator Mask and ViraCoat FFP3 Respirator mask, a new range of antiviral and antimicrobial personal protective equipment (PPE) masks that begin to kill SARS-CoV-2 and other viruses on contact. This is a major breakthrough in reducing risks, to staff and patients, of COVID-19, as there is a significant reduction in contamination risk during handling of masks while in use. They will also reduce costs, because there is no need to change masks multiple times per working shift. The product has gone through rigorous validation with Lancaster University and is now going into production in the UK.

The ViraCoat masks are impregnated with a novel and patented antiviral and antimicrobial coating that completely kills SARS-CoV-2 virus within minutes. The entire surface of the mask is covered in the antimicrobial coating, including the straps; this significantly reduces the risk of contamination during handling of the masks and therefore transfer of the virus to the wearer or patient, even if the mask is removed and replaced several times during a shift. Many hospitals report health workers changing masks between three and eight times throughout the course of the day as they move between patients or tasks. ViraCoat's COVID-19-eliminating masks could save millions of tons of masks ending up in landfill and our oceans every year. Less frequent replacement means a significant potential financial saving for public and private health buyers, and other high-volume users.

The masks are ergonomically shaped for comfort and have an adjustable nose bridge

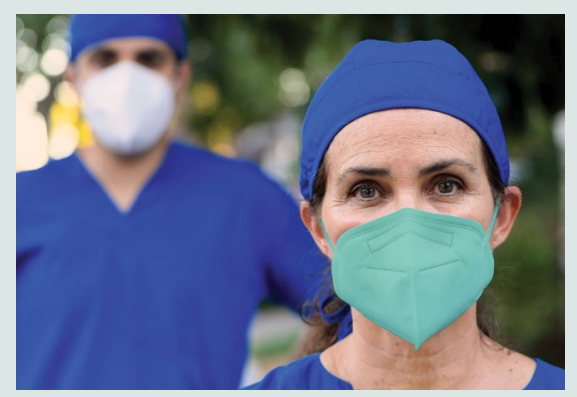

for an optimal seal to prevent contamination by infectious aerosols - tiny virus particles that linger in the air and are now widely accepted as a source of coronavirus infections not screened by surgical style masks. They are a distinctive green colour to easily differentiate from standard PPE. At this stage the ViraCoat antimicrobial coating is being applied to masks conforming to the filter-barrier requirements of EU Standard EN149:2001 + A1: 2009 type filtering efficiency standard to meet current regulations. However, the coating obviates the need for these types of barriers and new standards are being sought that will allow ViraCoat antimicrobial masks to be a lighter, more comfortable single layer, which can be repeatedly washed or sterilised.

The new masks, over a year in development, have undergone rigorous testing in the world-class biomedical facilities at Lancaster University.

ViraCoat is a subsidiary of ViraCorp, a group of biomedical research and development companies focused on developing viral defence products against the viral outbreaks, pandemics, and endemics that threaten world health; the ViraCoat range of masks is the first product to go into production. The masks will be in UK production at scale in October with the highest standards of quality control.

https://viracorp.global/

\section{A HAPPY TEAM IS A BETTER TEAM}
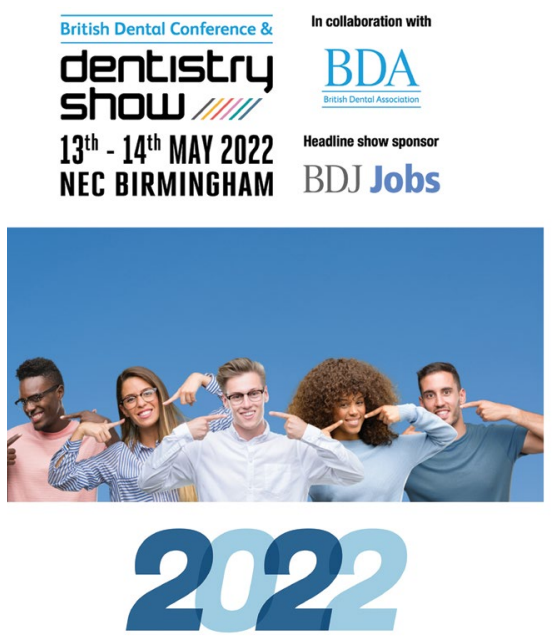

Happiness among staff at work is linked to increased productivity, enhanced teamwork and improved patient experiences. Help your team overcome the challenges of the past year by bringing a little joy to your practice.

A major enabler to job satisfaction is career development, so why not support your colleagues in their ambitions by taking the whole team to the British Dental Conference and Dentistry Show. Not only will there be education and enhanced CPD for all delivered by world-class speakers, but this will be accompanied by an extensive trade exhibition demonstrating the latest innovations to re-inspire delegates.

This will also be a great opportunity to enjoy some social time with the team and reconnect after a difficult few months. After all, a happy team is a better team.

The British Dental Conference and Dentistry Show will be held on Friday 21 and Saturday 22 May 2022, Birmingham NEC, co-located with DTS.

www.thedentistryshow.co.uk

\section{NO MORE GUESSWORK}

When the $\mathrm{x}$-ray film is inside the mouth it's difficult to know exactly where it is, especially when patients inadvertently move it with their tongue as they swallow. Dürr Dental's film holders remove the guesswork, so that the radiation hits the correct tooth, at the correct angle, hitting $100 \%$ of the film. No more retakes! Moreover, these holders have been developed specifically for use with phosphor plates, so they won't cause damage, unlike traditional ones designed for use with film.

To facilitate use, the holders have been colour-coded, which saves time when assembling. They are also available to buy as individual components, so that if an element gets lost, or eventually becomes worn, then only that component needs to be ordered. This saves money and has environmental benefits too.

Dürr's image plate holders can be used with any make of phosphor plate and will

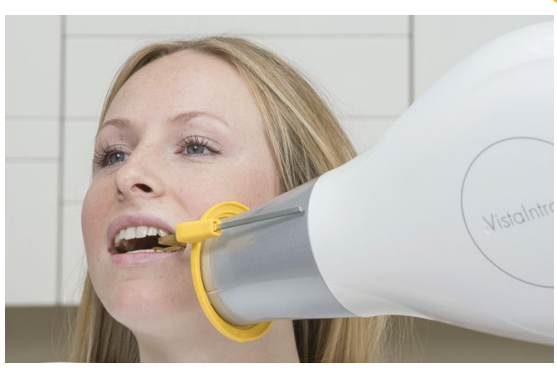

allow you to position the film exactly where you want it. They won't distort over time and offer the patient significantly improved radiation protection. 